PROCEEDINGS OF THE

AMERICAN MATHEMATICAL SOCIETY

Volume 138, Number 1, January 2010, Pages 367-373

S 0002-9939(09)10042-4

Article electronically published on August 20, 2009

\title{
CHERN SUBRINGS
}

\author{
MASAKI KAMEKO AND NOBUAKI YAGITA
}

(Communicated by Paul Goerss)

\begin{abstract}
Let $p$ be an odd prime. We show that for a simply connected semisimple complex linear algebraic group, if its integral homology has $p$ torsion, the Chern classes do not generate the Chow ring of its classifying space.
\end{abstract}

\section{INTRODUCTION}

Let $p$ be an odd prime. Let $h^{*}(-)$ be one of the $\bmod p$ cohomology $H \mathbb{Z} / p$, the cohomology $H \mathbb{Z}_{(p)}$ with coefficient $\mathbb{Z}_{(p)}$ and the Brown-Peterson cohomology $B P$ with $B P_{*}=\mathbb{Z}_{(p)}\left[v_{1}, v_{2}, \ldots\right]$. Let $G$ be a compact connected Lie group and $G(\mathbb{C})$ its complexification; that is, $G(\mathbb{C})$ is a complex linear algebraic group which is homotopy equivalent to the compact connected Lie group $G$. Considering a finite dimensional complex representation $\rho: G \rightarrow G L_{m}(\mathbb{C})$, we have Chern classes $c_{i}(\rho)$ in the cohomology $h^{*}(B G)$ of classifying space and the Chern subring $C h_{h}(G) \subset$ $h^{*}(B G)$, a subalgebra over $h_{*}$ generated by Chern classes, where $\rho$ ranges over all finite dimensional representations. If $G$ is one of the classical groups $S U(n)$, $\operatorname{Spin}(n)$ and $S p(n)$, the cohomology $h^{*}(B G)$ is generated by Chern classes and $h^{*}(B G)=C h_{h}(G)$ for an arbitrary odd prime $p$.

The case of the Brown-Peterson cohomology is particularly interesting in conjunction with the study of Chow rings of classifying spaces of complex linear algebraic groups defined by Totaro. In [To, Totaro considered the classifying space of the linear algebraic group $G(\mathbb{C})$ as a limit of algebraic varieties, defined the Chow ring for it and showed that the cycle map factors through the Brown-Peterson cohomology,

$$
C H^{*}(B G(\mathbb{C}))_{(p)} \rightarrow B P^{*}(B G) \otimes_{B P_{*}} \mathbb{Z}_{(p)} \rightarrow H^{\text {even }}\left(B G ; \mathbb{Z}_{(p)}\right),
$$

where $H^{\text {even }}\left(B G ; \mathbb{Z}_{(p)}\right)$ is the direct sum of $H^{2 i}\left(B G ; \mathbb{Z}_{(p)}\right)(i \geq 0)$. He also conjectured that the left homomorphism $C H^{*}(B G(\mathbb{C}))_{(p)} \rightarrow B P^{*}(B G) \otimes_{B P_{*}} \mathbb{Z}_{(p)}$ is an isomorphism. We may consider a Chern subring for the Chow ring $C H^{*}(B G(\mathbb{C}))$ as in the case of the above $C h_{h}(G)$.

In $\mathrm{Ka}-\mathrm{Ya}$ and $\mathrm{Vi}$, the Chow ring $C H^{*}\left(B P G L_{p}(\mathbb{C})\right)_{(p)}$ of the complex linear algebraic group $P G L_{p}(\mathbb{C})$, which is the complexification of the projective unitary

Received by the editors November 2, 2008, and, in revised form, May 7, 2009.

2000 Mathematics Subject Classification. Primary 55R40, 57T15, 20J06, 20J05.

Key words and phrases. Chow ring, Chern class, classifying space, motivic cohomology.

The authors are partially supported by the Japan Society for the Promotion of Science, Grantin-Aid for Scientific Research (C) 19540105, 20540061, respectively.

(C)2009 American Mathematical Society Reverts to public domain 28 years from publication 
group $P U(p)$, and related cohomology theories were computed, and it was shown that

$$
C H^{*}\left(B P G L_{p}(\mathbb{C})\right)_{(p)}=B P^{*}(B P U(p)) \otimes_{B P_{*}} \mathbb{Z}_{(p)}=H^{\text {even }}\left(B P U(p) ; \mathbb{Z}_{(p)}\right)
$$

through the cycle map above. In [Ka-Ya, Proposition 5.7], we showed similar results for $(p, G)=\left(3, F_{4}\right),\left(5, E_{8}\right)$. For $p=3$, the computation of the Brown-Peterson cohomology was done by Kono and Yagita in Ko-Ya, and Kono and Yagita showed that $x_{8}^{a}$ is not in the Chern subring unless $a$ is divisible by 2. In [Ta], Targa showed that $x_{2 p+2}^{a}$ in $C H^{*}\left(B P G L_{p}(\mathbb{C})\right)_{(p)}$, where $a \leq p-2$, is not in the Chern subring for an arbitrary odd prime $p$.

In this paper, we prove the following and generalize the above computation of Kono, Yagita and Targa. Let $Q_{i}$ be the Milnor operations of degree $2 p^{i}-1$ which acts on the $\bmod p$ cohomology of a space.

Theorem 1.1. For $(p, G)=(p, P U(p))$, let $x=Q_{0} Q_{1} x_{2}$ where $x_{2}$ is the generator of $H^{2}(B G ; \mathbb{Z} / p)=\mathbb{Z} / p$. For $(p, G)=\left(3, F_{4}\right),\left(3, E_{6}\right),\left(3, E_{7}\right),\left(3, E_{8}\right),\left(5, E_{8}\right)$, let $x=Q_{1} Q_{2} x_{4}$, where $x_{4}$ is the generator of $H^{4}(B G ; \mathbb{Z} / p)=\mathbb{Z} / p$. Then, $x^{a}$ is not in the Chern subring $C h_{H \mathbb{Z} / p}(G)$ unless a is divisible by $p-1$.

This theorem implies that if $x$ comes from the Chow ring through the cycle map, then the Chow ring is not generated by Chern classes. Recall that the motivic cohomology $H^{*, *^{\prime}}(B G(\mathbb{C}), \mathbb{Z} / p)$ contains $C H^{*}(B G(\mathbb{C})) / p$ as

$$
C H^{*}(B G(\mathbb{C})) / p=H^{2 *, *}(B G(\mathbb{C}), \mathbb{Z} / p) .
$$

Moreover, the motivic cohomology has the action of Milnor operations $Q_{i}$ where the degree of $Q_{i}$ is $\left(2 p^{i}-1, p^{i}-1\right)$. We refer the reader to Voevodsky's paper $\mathrm{VO}$ for motivic cohomology operations. If there exists an element $x_{4,3}$ in $H^{4,3}(B G(\mathbb{C}), \mathbb{Z} / p)$ corresponding to $x_{4}$ in $H^{4}(B G ; \mathbb{Z} / p)$, then $x=Q_{1} Q_{2}\left(x_{4,3}\right)$ is in the Chow ring

$$
C H^{p^{2}+p+1}(B G(\mathbb{C})) / p=H^{2 p^{2}+2 p+2, p^{2}+p+1}(B G(\mathbb{C}), \mathbb{Z} / p)
$$

and through the cycle map it maps to $x$ in Theorem 1.1. In [Ya, Lemma 9.6], Yagita proved that if $p x_{4} \in H^{4}\left(B G ; \mathbb{Z}_{(p)}\right)$ is a Chern class of some representation, then the element $x_{4,3}$ above exists. In $\mathrm{Sc}-\mathrm{Ya}$, Schuster and Yagita showed that for $(p, G)=\left(3, F_{4}\right), 3 x_{4}$ is the Chern class of the complexification of the irreducible representation of $F_{4}$. In this paper, by computing the Chern class of the adjoint representation of $E_{8}$, we prove the following proposition.

Proposition 1.2. For $(p, G)=\left(3, F_{4}\right),\left(3, E_{6}\right),\left(3, E_{7}\right),\left(3, E_{8}\right)$ and $\left(5, E_{8}\right)$, there exist a complex representation $\alpha$ of $G$ and $\gamma \in \mathbb{Z}_{(p)}^{\times}$such that the element $\gamma p x_{4} \in$ $H^{4}\left(B G ; \mathbb{Z}_{(p)}\right)$ is a Chern class $c_{2}(\alpha)$.

Thus, we have the following result on Chern subrings of Chow rings.

Theorem 1.3. For $(p, G)=(p, P U(p)),\left(3, F_{4}\right),\left(3, E_{6}\right),\left(3, E_{7}\right),\left(3, E_{8}\right)$ and $\left(5, E_{8}\right)$, the Chow ring $C H^{*}(B G(\mathbb{C}))_{(p)}$ is not generated by Chern classes.

Our results seem to support Totaro's conjecture since not only elements in $C h_{B P}(G)$ but also some elements that are not in the Chern subrings come from Chow rings $C H^{*}(B G(\mathbb{C}))$ for the above $(p, G)$.

In $\S 2$, we consider Chern classes of elementary abelian $p$-groups. In $\S 3$, we prove Theorem 1.1, In $\S 4$, we prove Proposition 1.2. Throughout the rest of this paper, we write $H^{*}(-)$ for $H^{*}(-; \mathbb{Z} / p)$ whenever the coefficient $\mathbb{Z} / p$ is clear from the context.

We thank François-Xavier Dehon for informing us of the work of Targa. 


\section{Chern Classes of elementary abelian $p$-Groups}

In this section, we investigate the total Chern class of a finite dimensional complex representation $\rho: A_{n} \rightarrow G L_{m}(\mathbb{C})$ of an elementary abelian $p$-group $A_{n}$ of rank $n$.

First, we recall the cohomology of $B A_{n}$. The mod $p$ cohomology of an elementary abelian $p$-group is a polynomial tensor exterior algebra

$$
\mathbb{Z} / p\left[t_{1}, \ldots, t_{n}\right] \otimes \Lambda\left(d t_{1}, \ldots, d t_{n}\right) .
$$

The elements $d t_{1}, \ldots, d t_{n} \in H^{1}\left(B A_{n}\right)$ correspond to the dual of the basis of $\pi_{1}\left(B A_{n}\right)=H_{1}\left(B A_{n}\right)$. The elements $t_{1}, \ldots, t_{n}$ are obtained from $d t_{1}, \ldots, d t_{n}$ by applying the Milnor operation $Q_{0}$. For the $\bmod p$ cohomology of a space, there exists an action of Milnor operations $Q_{0}, Q_{1}, Q_{2}, \ldots$ and reduced power operations $\wp^{0}=1, \wp^{1}, \wp^{2}, \ldots$ The action of Milnor operations on the mod $p$ cohomology of an elementary abelian $p$-group is given by

$$
Q_{i}\left(d t_{k}\right)=t_{k}^{p^{i}}, \quad Q_{i} t_{k}=0, \quad Q_{i}(x \cdot y)=Q_{i}(x) \cdot y+(-1)^{\operatorname{deg} x} x \cdot Q_{i}(y) .
$$

The action of reduced power operations is given by

$$
\wp^{i} d t_{k}=0, \quad \wp^{i} t_{k}=\left\{\begin{array}{ll}
t_{k}^{p} & (i=1) \\
0 & (i \geq 2),
\end{array} \quad \wp^{j}(x \cdot y)=\sum_{i=0}^{j} \wp^{i-j} x \cdot \wp^{j} y .\right.
$$

Second, we recall the invariant theory of finite general linear groups and special linear groups. The action of Milnor operations commutes with the action of the general linear group $G L_{n}(\mathbb{Z} / p)$ since the action of the general linear group on the mod $p$ cohomology comes from the one on the elementary abelian $p$-group $A_{n}$. For the sake of notational simplicity, we write $V_{n}$ for the subspace spanned by $t_{1}, \ldots, t_{n}$,

$$
V_{n}=\mathbb{Z} / p\left\{t_{1}, \ldots, t_{n}\right\}
$$

Let

$$
\begin{aligned}
& S M_{n}=H^{*}\left(B A_{n}\right)^{S L_{n}(\mathbb{Z} / p)}, \quad M_{n}=H^{*}\left(B A_{n}\right)^{G L_{n}(\mathbb{Z} / p),} \\
& S D_{n}=\mathbb{Z} / p\left[t_{1}, \ldots, t_{n}\right]^{S L_{n}(\mathbb{Z} / p)}, \quad D_{n}=\mathbb{Z} / p\left[t_{1}, \ldots, t_{n}\right]^{G L_{n}(\mathbb{Z} / p),}
\end{aligned}
$$

respectively. Kameko and Mimura Ka-Mi] gave a simpler description for $S M_{n}$, $M_{n}$ using Milnor operations. For the Dickson invariants $S D_{n}, D_{n}$ and the Mùi invariants $S M_{n}, M_{n}$ above, we refer the reader to [Ka-Mi] and its references. Let us define $c_{n, i}$ for $n=1, \ldots, n-1$ as follows: Consider the polynomial

$$
f_{n}(X)=\prod_{v \in V_{n}}(X+v)
$$

in $\mathbb{Z} / p\left[t_{1}, \ldots, t_{n}\right][X]$. We define $(-1)^{n-i} c_{n, i}$ to be the coefficient of $X^{p^{n-i}}$ in $f_{n}(X)$. We define $e_{n}$ by $e_{n}=Q_{0} \cdots Q_{n-1}\left(d t_{1} \cdots d t_{n}\right)$. Then, we have the following. For a ring $R$ and for a finite set $\left\{a_{1}, \ldots, a_{r}\right\}$, we denote by $R\left\{a_{1}, \ldots, a_{r}\right\}$ a free $R$-module with the basis $\left\{a_{1}, \ldots, a_{r}\right\}$.

Proposition 2.1. The following hold:

(1) $c_{n, 0}=e_{n}^{p-1}$.

(2) $f_{n}(X)=X^{p^{n}}-c_{n, n-1} X^{p^{n-1}}+\cdots+(-1)^{n} c_{n, 0} X$.

(3) $S D_{n}$ is a polynomial algebra $\mathbb{Z} / p\left[e_{n}, c_{n, n-1}, \ldots, c_{n, 1}\right]$.

(4) $D_{n}$ is also a polynomial algebra $\mathbb{Z} / p\left[c_{n, n-1}, \ldots, c_{n, 1}, c_{n, 0}\right]$. 
(5) $M_{n}$ is a free $D_{n}$-module

$$
D_{n}\left\{1, e_{n}^{p-2} d t_{1} \ldots d t_{n}, e_{n}^{p-2} Q_{i_{1}} \ldots Q_{i_{r}}\left(d t_{1} \cdots d t_{n}\right)\right\} \quad \text { and }
$$

(6) $S M_{n}$ is a free $S D_{n}$-module

$$
S D_{n}\left\{1, d t_{1} \ldots d t_{n}, Q_{i_{1}} \ldots Q_{i_{r}}\left(d t_{1} \cdots d t_{n}\right)\right\},
$$

where $0 \leq i_{1}<\cdots<i_{r} \leq n-1,1 \leq r \leq n-1$.

Third, we consider Chern classes. Any finite dimensional complex representation of an abelian group is a direct sum of 1-dimensional complex representations. (See Serre's book [Se, Theorem 9].) Therefore, the total Chern class $c(\rho)$ is a product of $c(\lambda)$ 's where $c(\lambda)=1+v, v \in V_{n}$. Thus, the Chern classes are in $\mathbb{Z} / p\left[t_{1}, \ldots, t_{n}\right]$ instead of $H^{*}\left(B A_{n}\right)$. Let us consider the total Chern class $c($ reg $)$ of the regular representation reg $: A_{n} \rightarrow G L_{p^{n}}(\mathbb{C})$. It is clear that $c(r e g) \in M_{n}$.

Proposition 2.2. It follows that

$$
c(r e g)=\prod_{v \in V_{n} \backslash\{0\}}(1+v)=1-c_{n, n-1}+\cdots+(-1)^{n} c_{n, 0} \in D_{n} .
$$

For a group $W$ acting on $V_{n} \backslash\{0\}$, we say that the action of $W$ is transitive on $V_{n} \backslash\{0\}$ if and only if for each $u, v$ in $V_{n} \backslash\{0\}$, there exists $w \in W$ such that $w u=v$. We investigate the total Chern class $c(\rho)$ when the image of the induced homomorphism $B \rho^{*}: H^{*}\left(B G L_{m}(\mathbb{C})\right) \rightarrow \mathbb{Z} / p\left[t_{1}, \ldots, t_{n}\right]$ is invariant under a certain group action.

Lemma 2.3. Let $\rho: A_{n} \rightarrow G L_{m}(\mathbb{C})$ be a complex representation of an elementary abelian p-group $A_{n}$ of rank $n$. Suppose that a subgroup $W$ of $G L_{n}(\mathbb{Z} / p)$ acts on $A_{n}$ in the obvious manner. Suppose that the total Chern class $c(\rho)$ is in $\mathbb{Z} / p\left[t_{1}, \ldots, t_{n}\right]^{W}$ and suppose that the action of $W$ on $V_{n} \backslash\{0\}$ is transitive. Then, $c(\rho)=c(r e g)^{a}$ for some $a \geq 0$.

Proof. Suppose that

$$
c(\rho)=\prod_{v \in V_{n} \backslash\{0\}}(1+v)^{\mu(v)} .
$$

The non-negative integer $\mu(v)$ is the divisibility of $c(\rho)$ by $1+v$. In other words, $c(\rho)$ is divisible by $(1+v)^{\mu(v)}$ but not divisible by $(1+v)^{\mu(v)+1}$. In order to prove the lemma, it suffices to show that $\mu(v)$ is a constant function of $v \in V_{n} \backslash\{0\}$. Suppose that $\mu(u)<\mu(v)$ for some $u, v \in V_{n} \backslash\{0\}$. Let $w \in W$ be an element such that $w v=u$. Then, since $w$ acts trivially on $c(\rho)$, we have

$c(\rho)=w c(\rho)=\prod_{v^{\prime} \in V_{n} \backslash\{0\}}\left(w\left(1+v^{\prime}\right)\right)^{\mu\left(v^{\prime}\right)}=\left(\prod_{v^{\prime} \in V_{n} \backslash\{0, v\}}\left(1+w v^{\prime}\right)^{\mu\left(v^{\prime}\right)}\right)(1+u)^{\mu(v)}$.

This implies that $\mu(u) \geq \mu(v)$, a contradiction. Hence, we have the desired result.

By Proposition 2.2 and Lemma 2.3, we have the following result:

Proposition 2.4. Let $G$ be a compact connected Lie group and let $A_{n}$ be an elementary abelian p-subgroup of $G$. Suppose that the Weyl group of $A_{n}$, that is, the quotient of the normalizer of $A_{n}$ in $G$ by the centralizer of $A_{n}$ in $G$, acts transitively on $V_{n} \backslash\{0\}$. Then, $B \eta^{*}\left(C h_{H \mathbb{Z} / p}(G)\right) \subset D_{n}$, where $\eta: A_{n} \rightarrow G$ is the inclusion of $A_{n}$ into $G$. 
We end this section by recalling the following fact:

Proposition 2.5. The action of $S L_{n}(\mathbb{Z} / p)$ on $V_{n} \backslash\{0\}$ is transitive for $n \geq 2$.

Thus, in order to prove Theorem 1.1, it suffices to show that there exists an elementary abelian $p$-subgroup $A_{n}$ whose Weyl group is $S L_{n}(\mathbb{Z} / p)$ and that $B \eta^{*}(x) \notin$ $D_{n}$. This is what we do in the next section.

\section{Chern Subrings}

In this section, we prove Theorem 1.1 by examining the cohomology of a nontoral elementary abelian $p$-subgroup of $G$. There exist non-toral elementary abelian $p$-subgroups in a compact connected Lie group if the integral homology of the Lie group has $p$-torsion. These non-toral elementary abelian $p$-subgroups and their Weyl groups are known for $(p, G)=(p, P U(n)),\left(3, F_{4}\right),\left(3, E_{6}\right),\left(3, E_{7}\right),\left(3, E_{8}\right)$, and $\left(5, E_{8}\right)$. We refer the reader to Andersen et al. A-G-M-V] and its references. In this paper, we use the following results for $(p, G)=(p, P U(p)),\left(3, F_{4}\right)$ and $\left(5, E_{8}\right)$ only:

Proposition 3.1. The following hold:

(1) For $(p, G)=(p, P U(p))$, there exists a non-toral elementary abelian $p$ subgroup $A_{2}$ of rank 2 such that its Weyl group in $G$ is the special linear group $S L_{2}(\mathbb{Z} / p)$.

(2) For $(p, G)=\left(3, F_{4}\right),\left(5, E_{8}\right)$, there exists a non-toral elementary $p$-subgroup $A_{3}$ of rank 3 such that its Weyl group in $G$ is the special linear group $S L_{3}(\mathbb{Z} / p)$.

Let $\eta: A_{n} \rightarrow G$ be the inclusion of a non-toral elementary abelian $p$-subgroup in $G$. In $[\mathrm{Ka}-\mathrm{Ya}$, we computed the image of the induced homomorphism

$$
B \eta^{*}: H^{*}(B G) \rightarrow S M_{n}
$$

for $(p, G)=(p, P U(p)), n=2$ and for $(p, G)=\left(3, F_{4}\right),\left(3, E_{6}\right),\left(3, E_{7}\right),\left(5, E_{8}\right)$, $n=3$. Since we wish to include the case $(p, G)=\left(3, E_{8}\right)$ in Theorem 1.1, instead of making use of the computation of the image of $B \eta^{*}$, we use the following result, which is also used in the computation of the image of $B \eta^{*}$ :

Proposition 3.2. The following hold:

(1) The induced homomorphism

$$
H^{2}(B P U(p)) \rightarrow S M_{2}^{2}=\mathbb{Z} / p\left\{d t_{1} d t_{2}\right\}
$$

is an isomorphism.

(2) For $(p, G)=\left(3, F_{4}\right)$ and $\left(5, E_{8}\right)$, the induced homomorphism

$$
H^{4}(B G) \rightarrow S M_{3}^{4}=\mathbb{Z} / p\left\{Q_{0}\left(d t_{1} d t_{2} d t_{3}\right)\right\}
$$

is an isomorphism.

Now, we prove Theorem 1.1 for $(p, G)=\left(3, E_{8}\right)$. As we mentioned at the end of the previous section, it suffices to show that $B \eta^{*}(x) \notin D_{3}$. There is a sequence of inclusions

$$
F_{4} \rightarrow E_{6} \rightarrow E_{7} \rightarrow E_{8}
$$

and the induced homomorphisms

$$
H^{4}\left(B F_{4}\right) \leftarrow H^{4}\left(B E_{6}\right) \leftarrow H^{4}\left(B E_{7}\right) \leftarrow H^{4}\left(B E_{8}\right)=\mathbb{Z} / p
$$

are isomorphisms. Recall that we denote the generator of $H^{4}\left(B E_{8} ; \mathbb{Z} / 3\right)$ by $x_{4}$. We define $x \in H^{26}\left(B E_{8} ; \mathbb{Z} / 3\right)$ by $x=Q_{1} Q_{2}\left(x_{4}\right)$. Since the induced homomorphism 
maps $x_{4}$ to $Q_{0}\left(d t_{1} d t_{2} d t_{3}\right)$ by Proposition 3.2 it maps $x$ to $e_{3}=Q_{0} Q_{1} Q_{2}\left(d t_{1} d t_{2} d t_{3}\right)$ in $S D_{3}$. Since $D_{3}$ is a polynomial algebra generated by $e_{3}^{p-1}, c_{3,1}$ and $c_{3,2}$ and since $S D_{3}$ is a polynomial algebra generated by $e_{3}, c_{3,1}, c_{3,2}$, it is clear that $e_{3}^{a}$ is in $D_{3}$ if and only if $a$ is divisible by $p-1$. Thus, we have Theorem 1.1 for $(p, G)=\left(3, E_{8}\right)$. Theorem 1.1 for the other $(p, G)$ 's can be proved in the same manner.

\section{Proof of Proposition 1.2}

In this section, we prove Proposition 1.2 by computing the second Chern class of the adjoint representation of the exceptional Lie group $\alpha: E_{8} \rightarrow S O(248)$. Similar computations were done in $\mathrm{Sc}-\mathrm{Ya}$ ] for the irreducible representation $F_{4} \rightarrow S O(26)$.

Since the induced homomorphisms

$$
H^{4}\left(B F_{4} ; \mathbb{Z}_{(3)}\right) \leftarrow H^{4}\left(B E_{6} ; \mathbb{Z}_{(3)}\right) \leftarrow H^{4}\left(B E_{7} ; \mathbb{Z}_{(3)}\right) \leftarrow H^{4}\left(B E_{8} ; \mathbb{Z}_{(3)}\right)=\mathbb{Z}_{(3)}
$$

are isomorphisms, if $3 x_{4}$ in $H^{4}\left(B E_{8} ; \mathbb{Z}_{(3)}\right)$ is a Chern class, so it is in $H^{4}\left(B G ; \mathbb{Z}_{(3)}\right)$ for $G=F_{4}, E_{6}, E_{7}$. So, it suffices to show the proposition for $G=E_{8}$.

Let $\alpha: E_{8} \rightarrow S O(248)$ be the adjoint representation of $E_{8}$. By the construction of the exceptional Lie group $E_{8}$ in $[\mathrm{Ad}$, there exists a homomorphism $\beta: \operatorname{Spin}(16) \rightarrow E_{8}$ such that the induced representation $\alpha \circ \beta$ is the direct sum of $\lambda_{16}^{2}: \operatorname{Spin}(16) \rightarrow S O(120)$ and $\Delta_{16}^{+}: \operatorname{Spin}(16) \rightarrow S O(128)$. See [Ad, Corollary 7.3] and Mi-Ni, p. 143]. Let $T^{8}$ be the maximal torus of $\operatorname{Spin}(16)$. Let $T^{1}$ be the first factor of $T^{8}$ and $\eta: T^{1} \rightarrow \operatorname{Spin}(16)$ the inclusion of $T^{1}$ into $\operatorname{Spin}(16)$. Denote by $R(G)$ the complex representation ring of $G$. The complexification of $\lambda_{16}^{2}$ corresponds to the second elementary symmetric function of $z_{1}^{2}+z_{1}^{-2}, \ldots, z_{8}^{2}+z_{8}^{-2}$ in $R\left(T^{8}\right)$ and the complexification of $\Delta_{16}^{+}$corresponds to $\sum_{\varepsilon_{1} \cdots \varepsilon_{8}=1} z_{1}^{\varepsilon_{1}} \ldots z_{8}^{\varepsilon_{8}}$ in $R\left(T^{8}\right)$,

where $\varepsilon_{r}= \pm 1$ for $r=1, \ldots, 8$.

So, the restriction of the complexification of $\lambda_{16}^{2}$ to $T^{1}$ corresponds to

$$
2^{2}\left(\begin{array}{l}
7 \\
2
\end{array}\right)+2\left(\begin{array}{l}
7 \\
1
\end{array}\right)\left(z_{1}^{2}+z_{1}^{-2}\right)=84+14\left(z_{1}^{2}+z_{1}^{-2}\right) \quad \text { in } R\left(T^{1}\right) .
$$

The restriction of the complexification of $\Delta_{16}^{+}$to $T^{1}$ corresponds to

$$
2^{6}\left(z_{1}+z_{1}^{-1}\right)=64\left(z_{1}+z_{1}^{-1}\right) \quad \text { in } R\left(T^{1}\right) .
$$

Therefore, the total Chern class of the complexification of $\alpha \circ \beta \circ \eta$ is

$$
\{(1+2 u)(1-2 u)\}^{14}\{(1+u)(1-u)\}^{64}=1-120 u^{2}+\cdots \in \mathbb{Z}[u]=H^{*}\left(B T^{1} ; \mathbb{Z}\right),
$$

where $u$ is the generator of $H^{2}\left(B T^{1} ; \mathbb{Z}\right)=\mathbb{Z}$. Since $120=2^{3} \cdot 3 \cdot 5$, the Chern class $c_{2}(\alpha)$ represents $\gamma p x_{4}$ for $p=3,5$ in $H^{4}\left(B E_{8} ; \mathbb{Z}_{(p)}\right)$, where $\gamma$ is a unit in $\mathbb{Z}_{(p)}$ and $x_{4}$ is the generator of $H^{4}\left(B E_{8} ; \mathbb{Z}_{(p)}\right)=\mathbb{Z}_{(p)}$. This completes the proof of Proposition 1.2

\section{REFERENCES}

[Ad] J. F. Adams, Lectures on exceptional Lie groups, Univ. of Chicago Press, Chicago, IL, 1996. MR1428422 (98b:22001)

[A-G-M-V] K. K. S. Andersen, J. Grodal, J. M. Møller, and A. Viruel, The classification of $p$-compact groups for $p$ odd, Ann. of Math. (2) 167 (2008), no. 1, 95-210. MR.2373153 (2009a:55012)

[Ka-Mi] M. Kameko and M. Mimura. Mùi invariants and Milnor operations, Geometry and Topology Monographs, 11, Geom. Topol. Publ., Coventry, 2007, 107-140. MR2402803 
[Ka-Ya] M. Kameko and N. Yagita, The Brown-Peterson cohomology of the classifying spaces of the projective unitary groups $\mathrm{PU}(p)$ and exceptional Lie groups, Trans. Amer. Math. Soc. 360 (2008), no. 5, 2265-2284. MR2373313 (2008m:55008)

[Ko-Ya] A. Kono and N. Yagita, Brown-Peterson and ordinary cohomology theories of classifying spaces for compact Lie groups, Trans. Amer. Math. Soc. 339 (1993), no. 2, 781-798. MR1139493 (93m:55006)

[Mi-Ni] M. Mimura and T. Nishimoto. On the Stiefel-Whitney classes of the representations associated with Spin(15), Geometry and Topology Monographs, 11, Geom. Topol. Publ., Coventry, 2007, 141-176. MR.2402804

[Se] J.-P. Serre, Linear representations of finite groups, translated from the second French edition by Leonard L. Scott, Springer, New York, 1977. MR0450380 (56:8675)

[Sc-Ya] B. Schuster and N. Yagita, Transfers of Chern classes in BP-cohomology and Chow rings, Trans. Amer. Math. Soc. 353 (2001), no. 3, 1039-1054 (electronic). MR.1804412 (2002b:55030)

[Ta] E. Targa, Chern classes are not enough. Appendix to: "On the cohomology and the Chow ring of the classifying space of $\mathrm{PGL}_{p}$ " by A. Vistoli, J. Reine Angew. Math. 610 (2007), 229-233. MR2359887 (2008k:14012)

[To] B. Totaro, The Chow ring of a classifying space, in Algebraic K-theory (Seattle, WA, 1997), 249-281, Proc. Sympos. Pure Math., 67, Amer. Math. Soc., Providence, RI, 1999. MR:1743244 (2001f:14011)

[Vi] A. Vistoli, On the cohomology and the Chow ring of the classifying space of $\mathrm{PGL}_{p}$, J. Reine Angew. Math. 610 (2007), 181-227. MR.2359886 (2008k:14011)

[Vo] V. Voevodsky, Reduced power operations in motivic cohomology, Publ. Math. Inst. Hautes Études Sci. 98 (2003), 1-57. MR2031198(2005b:14038a)

[Ya] N. Yagita, Applications of Atiyah-Hirzebruch spectral sequences for motivic cobordism, Proc. London Math. Soc. (3) 90 (2005), no. 3, 783-816. MR2137831 (2006d:57049)

Faculty of Contemporary Society, Toyama University of International Studies, TOYAMA 930-1292, JAPAN

E-mail address: kameko@tuins.ac.jp

Faculty of Education, Ibaraki University, Mito, Ibaraki, Japan

E-mail address: yagita@mx.ibaraki.ac.jp 